\title{
A MULTI-NODE APPROACH TO SIMULATE THIN COASTAL STRUCTURES IN THE SPH CONTEXT
}

\author{
Francesco Aristodemo ${ }^{1}$, Domenico Davide Meringolo ${ }^{2}$, Paolo Veltri ${ }^{3}$
}

\begin{abstract}
We propose an improvement in modeling solid boundary conditions for 2D weakly-compressible Smoothed Particle Hydrodynamics (SPH) simulations for cases in which the thickness of the body is small compared to the desired particle size and the fluid surrounds the body from more than one side. Specifically, the fixed ghost particles technique developed by Marrone et al. (2011), based on interpolation nodes located within the fluid domain, is here extended to a multi-node approach. The fluid domain is thus divided into various sub-areas and an interpolation node for the considered solid particle is associated to every sub-area. Consequently, the solid particles present an array of values interpolated at different sub-areas for the same physical quantity. When a fluid particle located in a specific region interacts with a multi-node fixed ghost particle, the last assumes the field values interpolated in the reference area through the associated node. The present modeling allows to adopt a coarser spatial resolution to model the same physical problem, resulting in a reduction of the computational cost. The proposed solid boundary treatment is applied to horizontal decks and perforated wall-caisson breakwaters subjected to regular waves. In this context, an automatic hybrid diffusive formulation is introduced in order to prevent shock waves during water impacts and preserve the hydrostatic pressure. The formulation is obtained by defining a variable parameter detecting the occurrence of relevant density gradients induced by fluid impacts, resulting in an automatic switch between the two formulations.
\end{abstract}

Keywords: Smoothed Particle Hydrodynamics; solid boundaries; pressure field; coastal structures with thin elements

\section{INTRODUCTION}

SPH actually represents the most most popular meshless Lagrangian particle model due to its capabilities in simulating complex physical processes and the interaction with structures. A relevant amount of modeling during the last years has been performed in the SPH framework to simulate a broad range of flow phenomena and their interaction with structures such as wave impacts (e.g., Meringolo et al., 2015), environmental problems (Aristodemo et al., 2010) and jets into water bodies (Aristodemo et al., 2015b). In the SPH context, the treatment of solid boundary conditions is a fundamental topic for several applications in the hydraulic field using this modeling. Over the years many different approaches have been presented, which have therefore resulted in a lack of a universal technique. One of the major issues related with the particle approaching a solid boundary is the truncation of the kernel function by the body profile, resulting in a rapid decrease of the interpolation accuracy. For this reason, most of these techniques have been addressed to overcome this drawback. A widely adopted approach to deal with solid contours is the ghost particles technique (Colagrossi and Landrini, 2003). In the ghost particles framework, the fluid particles approaching the solid boundary are mirrored in respect to the body profile, in a layer with size equal to the kernel radius. Marrone et al. (2011) proposed an enhanced version of this method, introducing fixed ghost particles. In this case, the ghost particles are fixed in the frame of reference of the body and the values attributed to these particles are calculated at their interpolation nodes located inside the fluid domain. The values associated to them are obtained from a Moving Least Square (MLS) interpolation over the fluid particles.

In this paper, the fixed ghost particle treatment (Marrone et al., 2011) is extended to model solid boundaries in which the thickness of the body is small and the fluid ambient surrounds the wall from more than one side, as in the case of objects immersed in water. Specifically, the numerical simulation of a thin structure leads to choose a small initial spatial resolution for the considered problem, resulting in an increase of the computational cost. This situation often occurs in the numerical reproduction of laboratory experiments. Indeed, physical models of coastal structures are often made by slender elements such as the decks of offshore platforms or the slotted walls of breakwaters. Here, multi-node fixed ghost particles are introduced to overcome this drawback. The single fixed ghost particle is characterized by more interpolation nodes located in the fluid mass, resulting in an array of specific flow quantities associated to them, instead of a unique value. When the fluid particle interacts with the solid one, the latter assumes the values from the node depending on the spatial position of the fluid particle in the computational domain.

Weakly-compressible SPH simulations are here performed using a diffusive correction introduced in the continuity equation to limit the occurrence of high frequency oscillation in the pressure field. In this

\footnotetext{
${ }^{1}$ Department of Civil Engineering, University of Calabria, Via P. Bucci, Cubo 42B, 87036 Arcavacata di Rende (CS), Italy

${ }^{2}$ Department of Civil Engineering, University of Calabria, Via P. Bucci, Cubo 42B, 87036 Arcavacata di Rende (CS), Italy

${ }^{3}$ Department of Civil Engineering, University of Calabria, Via P. Bucci, Cubo 42B, 87036 Arcavacata di Rende (CS), Italy
} 
context, an improvement is proposed by considering an automatic hybrid term between the diffusive formulations proposed by Molteni and Colagrossi (2009) and Antuono et al. (2010). In this case, a parameter detecting the occurrence of fluid impacts is introduced in order to switch between the two considered formulations.

\section{SPH MODEL}

\section{Governing equations}

The discrete governing equations of the flow evolution for the adopted SPH model read as (e.g., Meringolo et al., 2015):

$$
\left\{\begin{array}{l}
\rho_{i} \frac{D \mathbf{u}_{i}}{D t}=-\sum_{j}\left(p_{i}+p_{j}\right) \nabla_{i} W\left(\mathbf{r}_{i j}\right) V_{j}+\rho_{i} \mathbf{g}_{i}+\alpha h c_{0} \rho_{0} \sum_{j} \pi_{i j} \nabla_{i} W\left(\mathbf{r}_{i j}\right) V_{j} \\
\frac{D \rho_{i}}{D t}=-\rho_{i} \sum_{j}\left(\mathbf{u}_{j}-\mathbf{u}_{i}\right) \cdot \nabla_{i} W\left(\mathbf{r}_{i j}\right) V_{j}+D_{i} \\
p_{i}=c_{0}^{2}\left(\rho_{i}-\rho_{0}\right) \\
\frac{D \mathbf{r}_{i}}{D t}=\mathbf{u}_{i}+\epsilon_{X} \sum_{j}\left(\mathbf{u}_{j}-\mathbf{u}_{i}\right) W\left(\mathbf{r}_{i j}\right) V_{j}
\end{array}\right.
$$

where $r_{j i}=-r_{i j}=r_{j}-r_{i}, \mathbf{g}_{i}$ represents the mass force acting on the fluid, the symbols $\rho_{i}, p_{i}, \mathbf{u}_{i}$ denote the $i$-th particle density, pressure, velocity, and $\rho_{0}$ and $c_{0}$ are the initial density and sound speed, respectively. The numerical value of $c_{0}$ is chosen in order the density variations remain lower than $1 \% \rho_{0}$. The particle volume $V_{i}$ is a function of the particle mass $m_{i}$, taken constant during the flow evolution, and the density. The term $\pi_{i j}$ represents the viscous contribution in the momentum equation. As regards the involved SPH parameters, $\alpha$ controls the artificial viscosity in the momentum equation and $\epsilon_{X}$ refers to the correction of the position of particles. In system (1) a linear state equation links pressure and density. A Gaussian kernel, $W_{j}\left(\mathbf{r}_{i}\right)$, is used in the simulations, adopting $h / \Delta x=4 / 3$ in which $h$ is smoothing length and $\Delta x$ is the spatial resolution. Concerning the integration scheme, a 4th order Runge Kutta with frozen diffusive approach is adopted (e.g. Aristodemo et al., 2015a). The time step $\Delta t$ is calculated on the basis of restrictive conditions linked to the use of the diffusive, advective and viscosity terms. The diffusive term $D_{i}$ in the continuity equation will be examined in the next Section.

\section{Diffusive terms}

Weakly-compressible SPH models presents the drawback of generating high frequency oscillations on the pressure field. This behavior, associated to the acoustic component of the weak compressibility, leads to a poor description of the pressure field. Recently, different authors have proposed many variations of the diffusive correction to stabilize the solution and attain more reliable results (e.g., Antuono et al., 2010). In general, the diffusive term is expressed in the following form:

$$
D_{i}=\delta h c_{0} \sum_{j} \psi_{j i} \frac{\mathbf{r}_{j i} \cdot \nabla_{i} W_{j}\left(\mathbf{r}_{i}\right)}{\left|\mathbf{r}_{i j}\right|^{2}} V_{j}
$$

in which $\delta$ is a parameter defining the magnitude of the diffusive term, while the term $\psi_{j i}$ is evaluated in accordance with the considered formulation. In the case of the formulation by Molteni and Colagrossi (2009), the term $\psi_{j i}$ is expressed as the density difference between the considered fluid particles:

$$
\psi_{j i M}=2\left(\rho_{j}-\rho_{i}\right)
$$

A different modeling has been presented by Antuono et al. (2010), in which the diffusive term is corrected with renormalized density gradients, and achieve in this case a higher approximation of the diffusive process. For this formulation, called $\delta$-SPH, the term $\psi_{j i}$ is evaluated as:

$$
\psi_{j i A}=2\left(\rho_{j}-\rho_{i}\right)-\left[\langle\nabla \rho\rangle_{j}^{L}+\langle\nabla \rho\rangle_{i}^{L}\right] \cdot \mathbf{r}_{j i}
$$


The quantity $\langle\nabla \rho\rangle_{i}^{L}$ represents the renormalized density gradient defined as:

$$
\begin{aligned}
\langle\nabla \rho\rangle_{i}^{L} & =\sum_{j}\left(\rho_{j}-\rho_{i}\right) L_{i} \nabla_{i} W_{j}\left(r_{i}\right) V_{j} \\
L_{i} & =\left[\sum_{j} r_{j i} \otimes \nabla_{i} W_{j}\left(r_{i}\right) V_{j}\right]^{-1}
\end{aligned}
$$

Antuono et al. (2012) presented a detailed analysis of diffusive-type terms, showing that the Molteni and Colagrossi approach presents an unphysical upwards displacement of the fluid particles near the free surface due to the inaccuracy near the free surface. This behavior is no longer encountered in the $\delta$-SPH scheme, as a result of the presence of the renormalized density gradients. Another issue related to the former models is the loss of potential energy, while the latter model preserves the hydrostatic solution. A different situation is encountered when high velocities and rapid changes in the fluid domain occur. In these cases, the model by Antuono et al. (2010) shows the occurrence of unphysical traveling sound waves after the impact with a solid boundary.

\section{Automatic hybrid diffusive term}

To overcome the limitations induced by the modeling of a pressure field characterized by a slow dynamic (absence of fluid-fluid and fluid-solid impacts) and a successive faster dynamics (violent impacts), Aristodemo et al. (2015a) have proposed a hybrid formulation between the Molteni and Colagrossi and Antuono et al. formulations. Specifically, a tuning parameter $\beta(r)$ was introduced in the formulation by Antuono et al. (2010), that activates or deactivates the renormalized density gradients as a function of the position of the particles inside the fluid domain. In this way, if a certain part of the domain is expected to be characterized by violent impacts would be implemented with $\beta(r)=0$, recovering therefore the Molteni and Colagrossi formulation, while for the remaining parts of the fluid domain it would be $\beta(r)=1$, recovering the Antuono et al. formulation. The result is that the portions of the fluid domain in which the specific diffusive formulation is acting have to be defined a priori and does not take into account for the instantaneous variation of the field values.

In this context, an improvement of the modeling is introduced by considering a tuning parameter that detects the occurrence of fluid impacts and automatically switches between the two formulations. In the weakly compressible context, the occurrence of fluid-fluid or fluid-solid impacts implies a spatial variation of pressure or, equivalently, density between the involved interacting particles. Since $\Delta \rho<1 \% \rho_{0}$, it results to be more convenient to consider the $\beta$-parameter as a function of the differences in density between the two interacting fluid particles, $\beta\left(\Delta \rho_{i j}\right)$. Therefore, the automatic hybrid diffusive term for $\psi_{i j H}$ becomes:

$$
\psi_{j i H}=2\left(\rho_{j}-\rho_{i}\right)-\left[\beta\left(\Delta \rho_{i j}\right)\langle\nabla \rho\rangle_{j}^{L}+\beta\left(\Delta \rho_{i j}\right)\langle\nabla \rho\rangle_{i}^{L}\right] \cdot \mathbf{r}_{j i}
$$

in which $\beta\left(\Delta \rho_{i j}\right) \in[0,1]$. In the case when $\beta\left(\Delta \rho_{i j}\right)=0$, the Molteni and Colagrossi (2009) formulation is recovered, while the Antuono et al. (2010) formulation is recovered when $\beta\left(\Delta \rho_{i j}\right)=1$. For values in the range $0<\beta\left(\Delta \rho_{i j}\right)<1$, a transition (or hybrid zone) between the two formulations is obtained. It is noticed that, in the case of free surface flows, and when fluid particles are located at different heights, there is a difference in density only due to the hydrostatic compression of the particles. This static quantity has to be subtracted because it does not count in hydrodynamic contribute. For a particle located at a water depth $d_{i}$ and considering the linearized state equation (see system 1), the value of the density in hydrostatic conditions results to be:

$$
\rho_{i s}=\rho_{0}+\frac{\rho_{0} g}{c_{0}^{2}} d_{i}
$$

The variation of density between the two interacting particle only due to the hydrodynamic contribution results, therefore, to be:

$$
\Delta \rho_{i}=\rho_{i}-\rho_{i s}=\rho_{i}-\rho_{0}-\frac{\rho_{0} g}{c_{0}^{2}} d_{i}
$$

If the water depth is $d_{i}=z_{\max }-z_{i}$, with $z_{\max }$ the dynamic free surface level assumed to be the same for the two particles, the differences in density between two particles only due to hydrodynamic contribute (water impacts) is:

$$
\Delta \rho_{i j}=\left|\rho_{i}-\rho_{j}+\frac{\rho_{0} g}{c_{0}^{2}}\left(z_{i}-z_{j}\right)\right|
$$


where the absolute value is used to incorporate the conditions $\Delta \rho_{i}>\Delta \rho_{j}$ and $\Delta \rho_{j}>\Delta \rho_{i}$.

The most extreme condition under weakly compressible assumption is that the $i$-th particle shows a value $\rho_{i}=\rho_{0}+1 \% \rho_{0}$ while the $j$-th particle a value $\rho_{j}=\rho_{0}-1 \% \rho_{0}$. The maximum difference in particles density is therefore equal to $\Delta \rho_{i j}=2 \% \rho_{0}$ and a dependence for $\beta\left(\Delta \rho_{i j}\right)$ has to be defined in the range $\Delta \rho_{i j} \in\left[0,2 \% \rho_{0}\right]$. Referring to Fig. 1, for small differences of density between the particles, the value of $\beta$ is equal to 1 and the $\delta$-SPH formulation is enforced. In this case, these variations of density are only associated to slow dynamics phenomena. The upper limit of this range is defined by the value $\Delta \rho_{A}$, in which the acoustic component starts to appear in the flow field. In the second range, the hybrid formulation takes place, in which the $\beta$-parameter assumes a linear variation between 1 , in correspondence to $\Delta \rho_{A}$, and 0 , in correspondence to $\Delta \rho_{M}$. In this range, high frequency noise starts to rise in the pressure field but it is still not relevant. For values higher than $\Delta \rho_{M}$, the parameter $\beta$ is set equal to 0 and the Molteni and Colagrossi formulation is enforced. In this case, significant variations in the density field due to the presence of fast dynamics and water impacts are encountered in the pressure field.

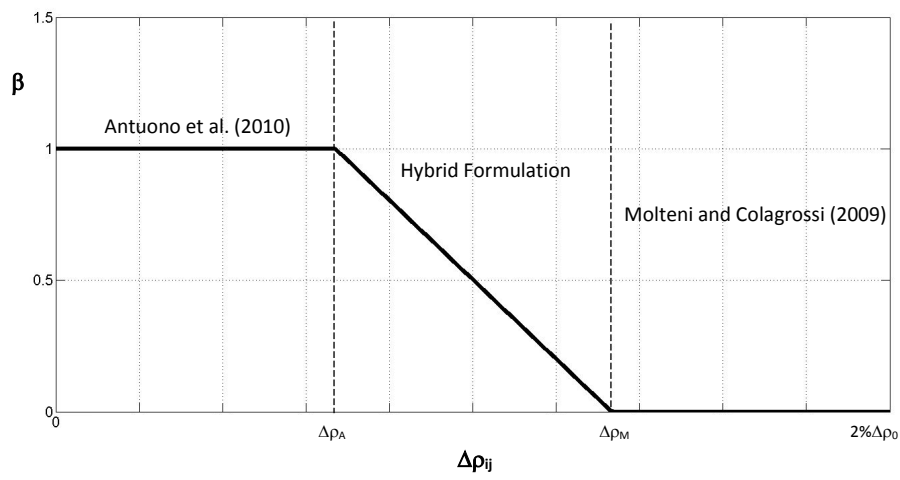

Figure 1: Variation of $\beta$-parameter as a function of the dynamic density difference between the fluid particles.

The values of $\Delta \rho_{i j}$ in which the transition between the different formulations occurs are here heuristically fixed to $\Delta \rho_{A}=0.3 \% \rho_{0}$ and $\Delta \rho_{M}=0.6 \% \rho_{0}$.

\section{SOLID BOUNDARY CONDITIONS}

In the fixed ghost particles framework (Marrone et al., 2011), the ghost particles are fixed in the frame of reference of the solid and are created only once at the beginning of the simulation with a regular distribution. The fixed ghost particles cover a body region with size equal to the width of the kernel support radius. In this way, as particles of the fluid domain approach the solid boundary, they will always find neighboring interacting particles and the accuracy related to kernel truncation is not compromised. For a support kernel with a radius equal to $3 h$ this would result in a layer formed by four lines of fixed ghost particles, equispaced with the initial resolution $\Delta x$. The field values associated to the fixed ghost particles are evaluated through a MLS interpolation performed in correspondence to the interpolation node.

\section{Multi-node fixed ghost particles}

In the fixed ghost particles approach, as previously described, it is necessary to cover a distance equal to the length of the kernel radius. For a problem in which the fluid mass is present at both sides of a solid boundary, to correctly enforce boundary conditions, four lines of fixed ghost particles interacting with fluid particles in the left side and four lines of fixed ghost particles interacting with fluid particles in the right side are necessary. This approach results in a layer consisting of eight lines of fixed ghost particles (case of kernel $3 h$ ). This situation does not represent any difficulty if the width of the solid body is big enough, but problems may arise if the structure is thin. Since the adopted initial spatial resolution can be often driven by the presence of thin solid elements, a high resolution is necessary even if it leads to disadvantages in terms of the computational time of the simulation. To overcome the problem, the CPU time can be reduced for example through Graphics Processing Units (e.g., Domínguez et al., 2013). The above solution could result in efforts in the implementation of the SPH code. 
A different solution can be obtained if, at every fixed ghost particle representing a thin structure, more interpolation nodes are associated, resulting in a multi-node fixed ghost particle approach. Thus the fluid domain is divided into sub-areas $\Omega_{k}$ and an interpolation node for the reference solid particle is associated to every sub-area. Consequently, the fixed ghost particles present an array of values interpolated at different sub-areas for the same physical quantity. When a fluid particle located in a region $\Omega_{k}$ interacts with a multinode fixed ghost particle, the last assumes the field values interpolated in the reference area $\Omega_{k}$ through the $k$-th node. In Fig. 2 a general case of the use of the multi-node approach is sketched, where the limits between the sub-regions are represented by dashed lines. For a correct modeling, the solid particles located away from the ends of the slender structure are characterized by only two nodes since the interaction with fluid particles occurs only from two sides, while the solid particles located on the ends, being surrounded by the fluid mass, are characterized by three interpolation nodes, with a resulting subdivision into three regions.

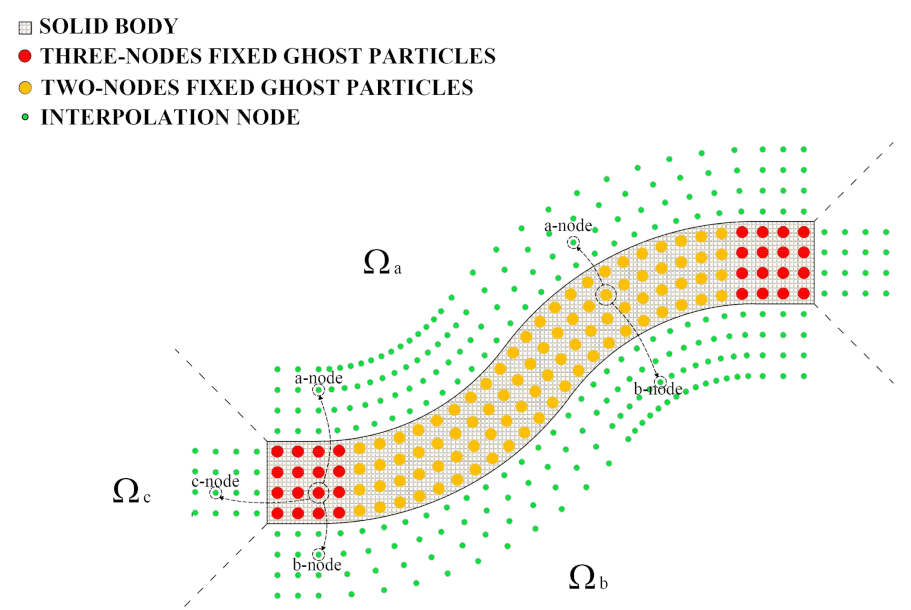

Figure 2: Sketch of multi-node fixed ghost particles with interpolation nodes for a solid body.

In Fig. 3 the differences in modeling a solid object with fixed ghost particles and multi-node fixed ghost particles are illustrated. With reference to the example in the right sketch in Fig. 3, the coordinates of the $k$-th node $\left(x_{k n}, z_{k n}\right)$ of the fixed ghost particles with coordinates $\left(x_{i}, z_{i}\right)$ are determined as follows:

$$
\begin{cases}a_{\text {node }}: & x_{a n}=x_{l}-\left(x_{i}-x_{l}\right), \quad z_{a n}=z_{i} \\ b_{\text {node }}: & x_{b n}=x_{r}+\left(x_{r}-x_{i}\right), \quad z_{b n}=z_{i} \\ c_{\text {node }}: & x_{c n}=x_{i}, \quad z_{c n}=z_{o}+\left(z_{o}-z_{i}\right)\end{cases}
$$

Moreover, it is possible to observe that the interpolation nodes of the solid particles located on the bisectors of the edges of the body are mirrored along the projection of the bisectors in the fluid domain using the classical fixed ghost particles framework (see left sketch in Fig. 3). In the multi-node approach, the node is instead always mirrored with the respect of the body profile. The transition between sub-areas occurs along the projection of the bisectors characterized by different interpolation nodes.

For 2D problems, the number of total particles required by the proposed multi-node technique is, in the case of a thin object driving the resolution, about $(1 / 2)^{2}=1 / 4$ the number of total particles requested by the use of the fixed ghost particles. It is interesting to notice that, in the case of extension to 3D simulations, the multi-node approach would result in a number of total particles that is approximatively $(1 / 2)^{3}=1 / 8$ the number of total particles needed with the classical approach. For 3D problems, the computational time saving would be quite higher than that obtained in the $2 \mathrm{D}$ cases.

\section{NUMERICAL RESULTS}

In this section, the proposed hybrid diffusive SPH model with an associated treatment of solid boundaries through multi-node fixed ghost particles is applied to study the interaction between regular waves and 


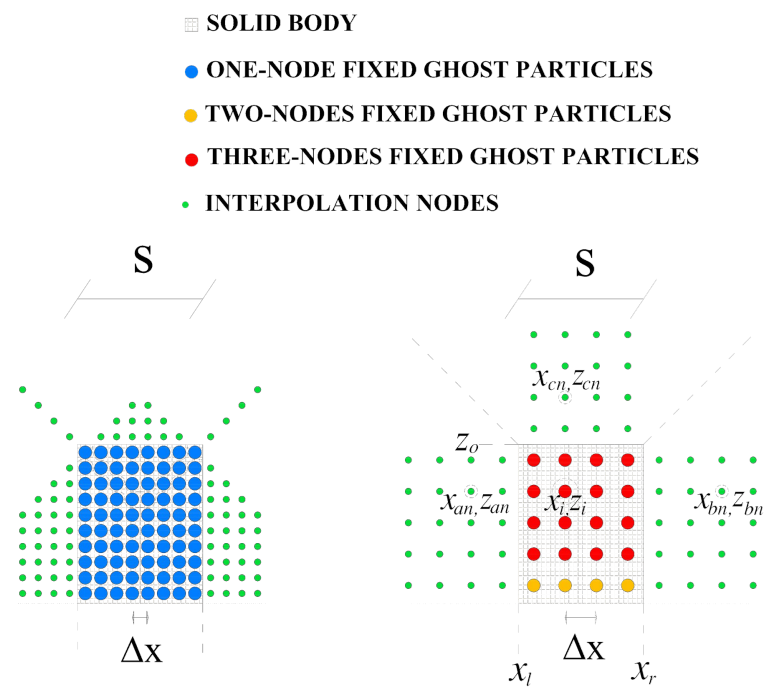

Figure 3: Differences between classical fixed ghost particles approach (left sketch) and multi-node approach (right sketch) in modelling a solid boundary (the value of $\Delta \mathbf{x}$ represents the minimal resolution necessary to model the body characterized by a width $s$ ).

coastal structures. Firstly, the flow impact around a horizontal deck under is studied. Afterward, the attention is paid to the evaluation of the dynamic pressures at a fully-perforated wall-caisson breakwater. The empirical parameters involved in the SPH equations are set as: $\alpha=0.01, \epsilon_{X}=0.25$ and $\delta=0.1$.

\section{Wave interaction with a horizontal deck}

This test refers to a horizontal deck placed above the Still Water Level (SWL) and subjected to water waves. As shown in Fig. 4, the adopted computation domain is characterized by a length of the wave flume $L_{c}=9 \mathrm{~m}$, a water depth $d=0.65 \mathrm{~m}$ and a distance between wave paddle and leading edge of the deck $x_{d}=2 \mathrm{~m}$. The length of the horizontal deck is $L_{d}=0.61 \mathrm{~m}$ and its height is $H_{d}=0.0115 \mathrm{~m}$, while the distance of its upper part from the SWL is $h_{d}=0.0525 \mathrm{~m}$. The deck is modeled by a combination of single and multi-node fixed ghost particles with corresponding interpolation nodes. The left wall of the channel is modeled by moving fixed ghost particles and implemented with the sinusoidal time law in order to simulate a piston-type wavemaker for wave generation. It is worth noting that mobile interpolation nodes follow the solid boundary condition simulating the wave paddle. The other solid boundaries are enforced using one-node fixed ghost particles. The adopted spatial resolution for the multi-node fixed particles is driven by the small height of the plate, so that $\Delta x=H_{d} / 4=0.002875 \mathrm{~m}$. Second-order Stokes waves are generated in the channel and checked by the number of wave harmonics in the spectral density of the surface elevation (e.g., Aristodemo et al., 2010). An amplitude of the stroke equal to $S_{0} / 2=0.25 \mathrm{~m}$ and a wave period $T=2$ $\mathrm{s}$ is imposed to the piston-type wavemaker.

In order to test the proposed boundary technique, the numerical results deduced from the present SPH model are compared with another SPH-based model developed by Gómez-Gesteira et al. (2005). A less refined spatial resolution $\Delta x=0.025 \mathrm{~m}$ with respect to the present SPH simulation was adopted by GómezGesteira et al. (2005). With reference to five significant time instants of the wave impact at the deck ( $t=$ $3.15 \mathrm{~s}, 3.27 \mathrm{~s}, 3.39 \mathrm{~s}, 3.45 \mathrm{~s}$ and $3.53 \mathrm{~s}$ ), Fig. 5 shows the particle positions (left panel) and the associated horizontal velocity field and streamlines (right panel) simulated through the proposed SPH solver. In the first selected time instant $(t=3.15 \mathrm{~s})$, the incoming wave reaches about the front face of the deck and a portion of the flow arrives at about the half of the lower zone of the deck, as observed by Gómez-Gesteira et al. (2005). The largest velocities appear at the wave crest and, due to the presence of the deck, in its lower zone. In the second frame $(t=3.27 \mathrm{~s})$, the wave crest overcomes the upper part of the deck with an increase in its steepness while the flow under the deck reaches its rear part where relevant velocities appear (Gómez-Gesteira et al., 2005). For $t=3.39 \mathrm{~s}$, the wave over the deck arrives at the rear of the deck while beyond it a breaking wave process induced by the flow past under the deck is noticed. A forward shift of the above mentioned physical processes are highlighted in the successive time window $(t=3.45 \mathrm{~s})$ in 


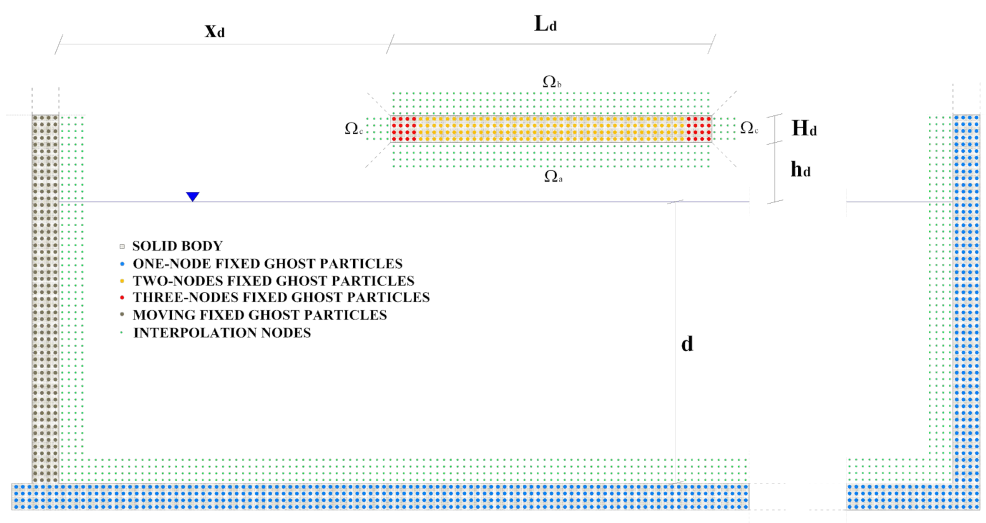

$\mathbf{L}_{\mathbf{c}}$

Figure 4: Sketch of solid boundary conditions modeled by a combination of single-node and multi-node fixed ghost particles and corresponding interpolation nodes for the case of the horizontal deck

which the water completely surrounds the involved horizontal structure. The formation of a vertical jet as a result of the interaction between the flows propagating upper and lower the deck is evident at the successive considered time $t=3.53 \mathrm{~s}$.

Moreover, the comparisons between the present SPH model and that developed by Gómez-Gesteira et al. (2005) are also performed in terms of time variation of pressure acting on the rear of the deck for the effect of the incoming wave motion, as described in Fig. 6 for a significant time window ranging from 3.15 s to $3.53 \mathrm{~s}$. On the basis of the model of Gómez-Gesteira et al. (2005), the above quantity was calculated by a spatial average of the instantaneous pressure at the two last solid particles of the deck, while it is here determined by the values of pressures located at the body profile belonging to the height of the rear deck. In the present simulation, the neighbor fluid particles are spatially averaged within a square support area with a length equal to $H_{d}$. In this case, the pressure field is characterized by the presence of only dynamic contribution since the deck has been placed above the SWL. Although the spatial resolution and the evaluation of the pressure at the rear of the deck using the SPH based-models is quite different, a general agreement on the behavior of the pressure can be observed. This is particularly evident for the peak value which appear close to the significant time instant $t=3.27 \mathrm{~s}$ where the flow upper the deck interacts with its rear part. After the maximum value of the pressure, a secondary peak simulated by the present SPH model proves to be forward shifter with respect to that simulated by Gómez-Gesteira et al. (2005) as well as the values of relative minimum of the pressure. It can be noticed that non-linear effects deduced from the adopted SPH solver could be associated to the more refined resolution adopted in space-time domain with respect to that applied to Gómez-Gesteira et al. (2005).

\section{Wave interaction with a perforated breakwater}

SPH simulations are here performed for the case of a fully perforated breakwater subjected to regular waves propagating along a plane channel. Concerning the geometry of the computational domain, the length of the wave flume is $L_{c}=4 \mathrm{~m}$, the water depth is $d=0.4 \mathrm{~m}$ and the chamber width is $B=0.54 \mathrm{~m}$. The height and the period of the incident waves are respectively $H=0.1 \mathrm{~m}$ and $T=1.15 \mathrm{~s}$, resulting in second-order Stokes wave trains at intermediate water depths.

To model the thin perforated wall of the caisson, multi-node fixed ghost particles are employed with the related interpolation nodes, as described in Fig. 7. Ghost particles with two or three nodes are adopted as a function of their position along the perforated wall. The spatial resolution used for the multi-node fixed ghost particles simulations has been driven by the width of the slotted wall, $s$, resulting in $\Delta x=s / 4=$ $0.0045 \mathrm{~m}$. For this test case it is also analyzed the tame saving due to the implementation of the multi-node approach, compared to the classical one. In the specific, the simulation of $10 \mathrm{~s}$ takes a CPU time of about $9 \mathrm{~h}$ using a single $3.4 \mathrm{GHz}$ Intel(R) i7-3770 core with 8GB RAM. Conversely, the use of classical fixed ghost particles requires a spatial resolution equal to $\Delta x=s / 8=0.00225 \mathrm{~m}$, as described in Fig. 3, resulting in a total CPU time equal to $44 \mathrm{~h}$ with the same computational strategies and machine. The simulation 

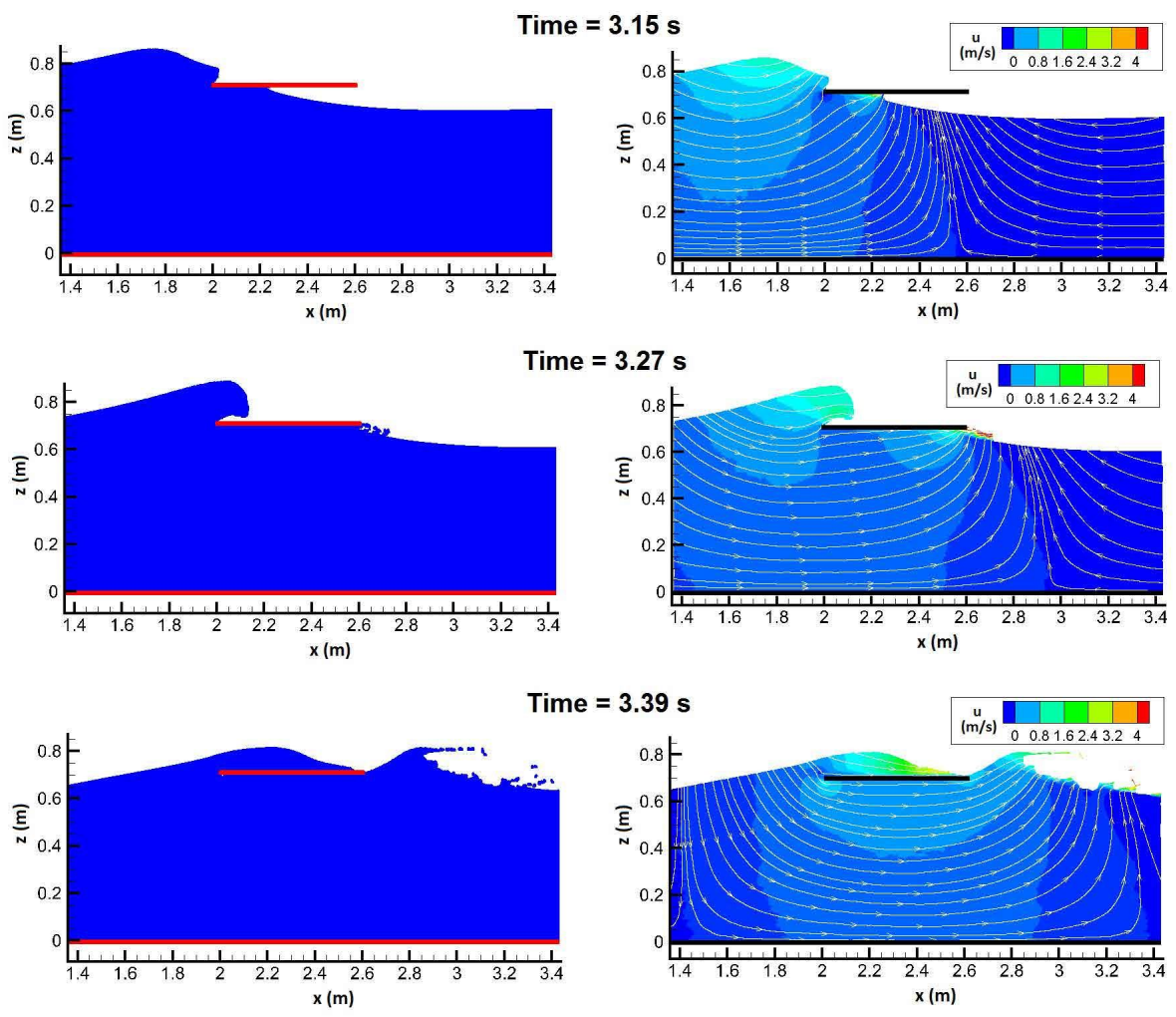

Time $=3.39 \mathrm{~s}$
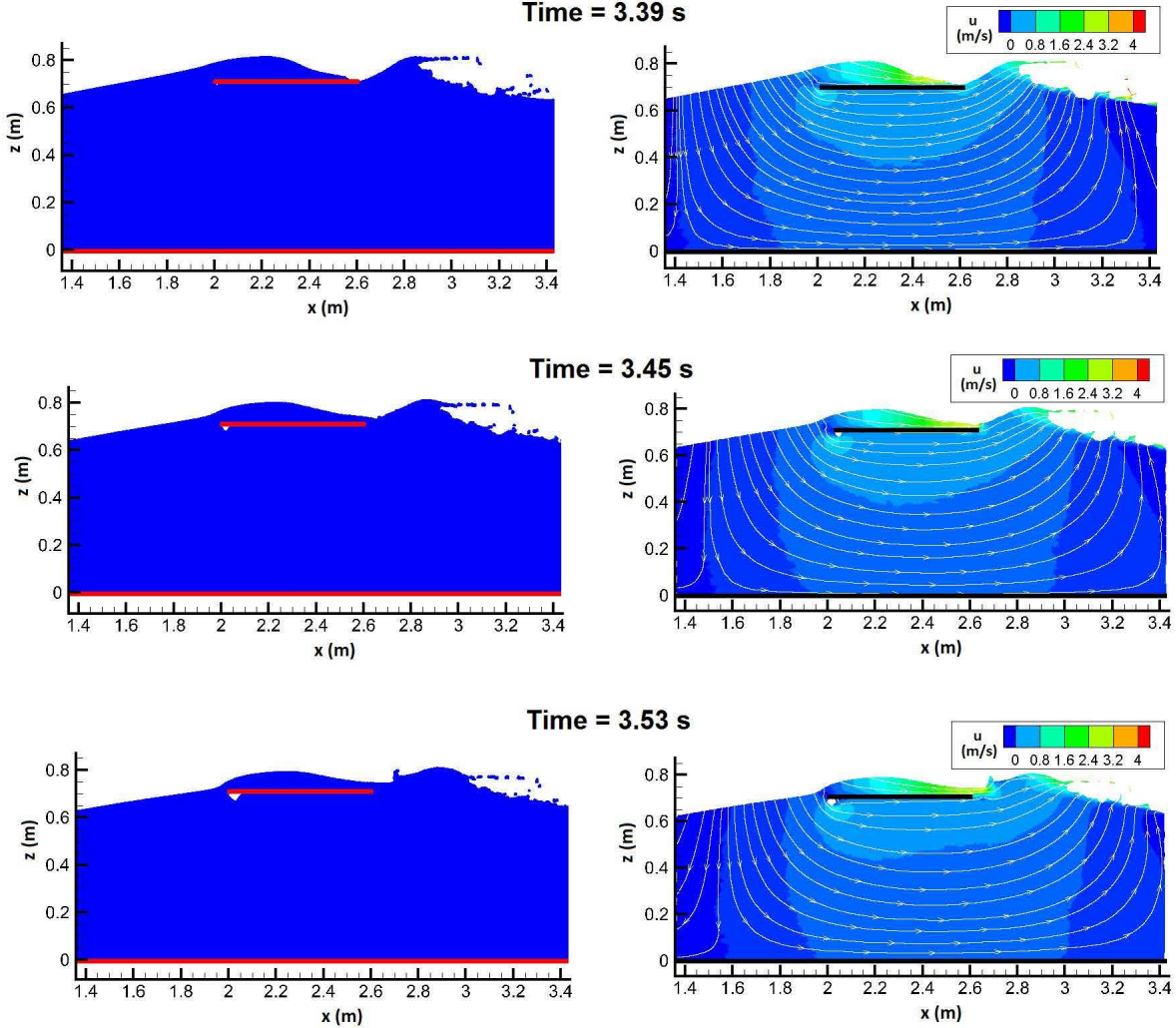

Figure 5: Particle positions (left panel) and associate horizontal velocity field and streamlines (right panel) simulated through the present SPH model at $t=3.15 \mathrm{~s}, 3.27 \mathrm{~s}, 3.39 \mathrm{~s}, 3.45 \mathrm{~s}$ and $3.53 \mathrm{~s}$. 


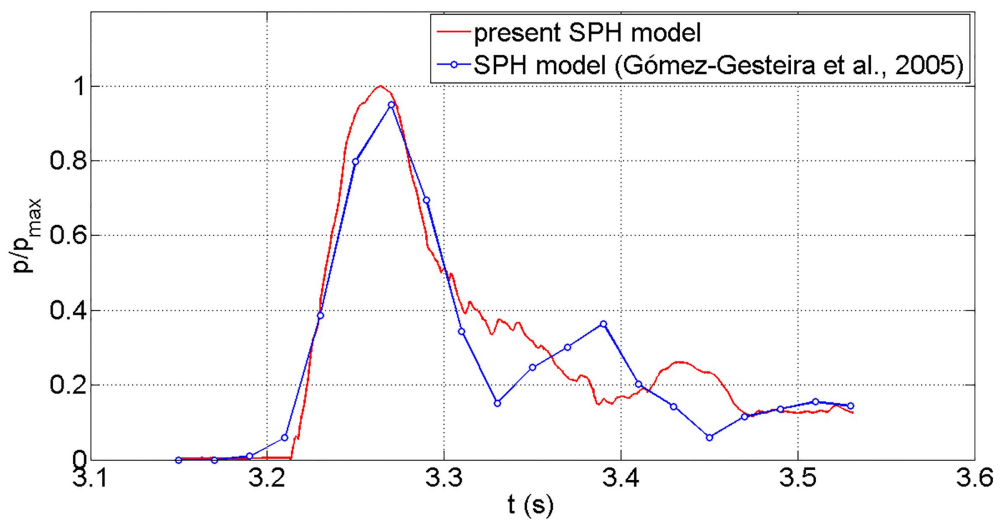

Figure 6: Time variation of pressure at the rear of the deck: comparison between present SPH model and that referred to Gómez-Gesteira et al. (2005).

with multi-node fixed ghost particles gives a reduction of the CPU time of about $79.5 \%$ with respect to the application of one-node fixed ghost particles. It is worth noting that in the standard implementation the spatial resolution is only driven by the modelling of the thin wall and it does not lead to an effective improvement in the accuracy of the numerical results. In order to provide a more detailed examination of the computational demand, two simulations performed with classical and multi-node fixed ghost particles at the identical spatial resolution were performed. In this case, the CPU time for the multi-node simulation resulted to be about $2 \%$ longer than that with classical fixed ghost particles. This slight difference is due to the fact that the multi-node treatment requires a half number of solid particles to model the thin wall and supplementary switches to compute the field values, resulting in a really small influence on the CPU time. In any case, this result is referred to this specific application, the difference in the computational demand being dependent on the number of total particles and on the number of multi-node fixed ghost particles.

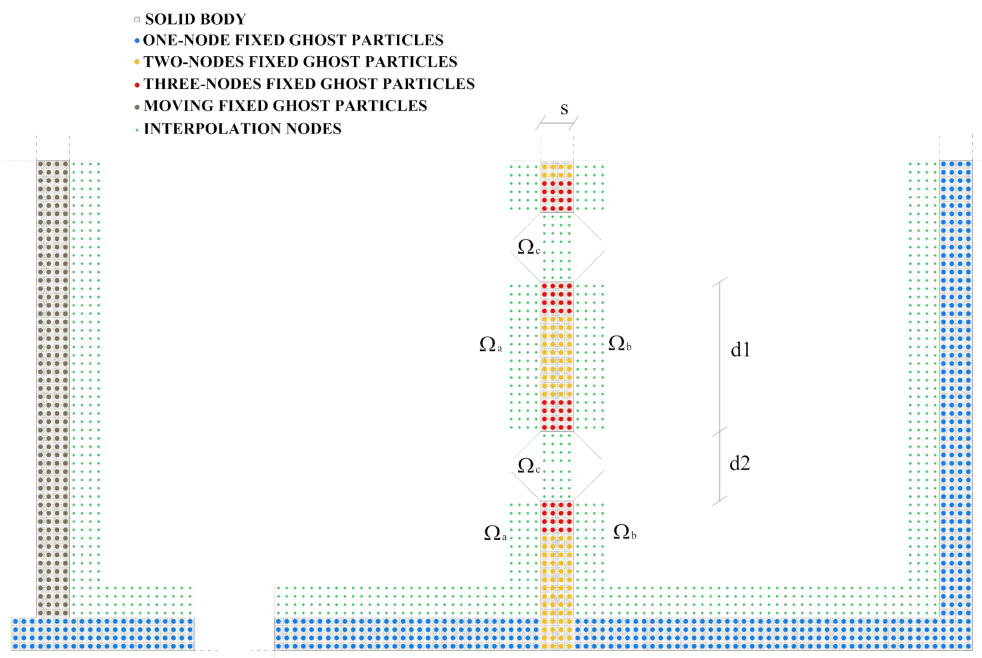

Figure 7: Sketch of solid boundary conditions modeled by a combination of single-node and multi-node fixed ghost particles and corresponding interpolation nodes for the case of the fully perforated breakwater (the width of the front wall is $s=0.018 \mathrm{~m}$, the height of the solid parts is $d \mathbf{1}=0.12 \mathrm{~m}$ and the height of the holes is $d \mathbf{2}=\mathbf{0 . 0 4} \mathbf{m})$.

In Figs. 8 and 9 two significant time instants of SPH simulations in which a wave interacts with the fully perforated breakwater are displayed. In these characteristic frames, pressure and horizontal velocity fields are shown. The considered two instants correspond to the maximum dynamic pressure occurring at the SWL for the front and rear faces, respectively. Indeed, the initial wave impact on the front wall and, 
successively, to the back wall occurs within a half wave cycle or, in other words, before the passage of the wave trough in correspondence with the perforated front wall.
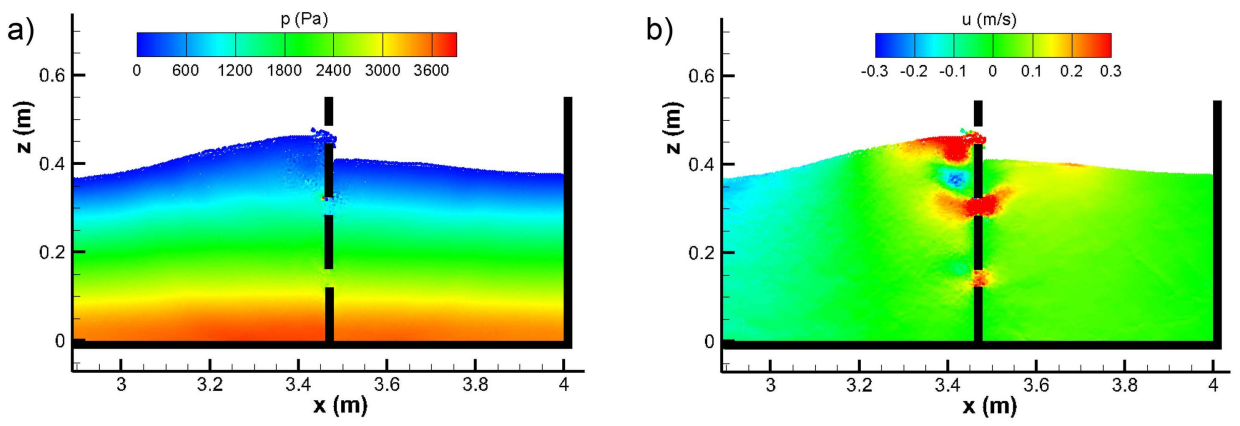

Figure 8: Frame of the SPH simulation in which the maximum dynamic pressure is encountered at the front face in correspondence to the SWL. a) pressure field, b) horizontal velocity field.
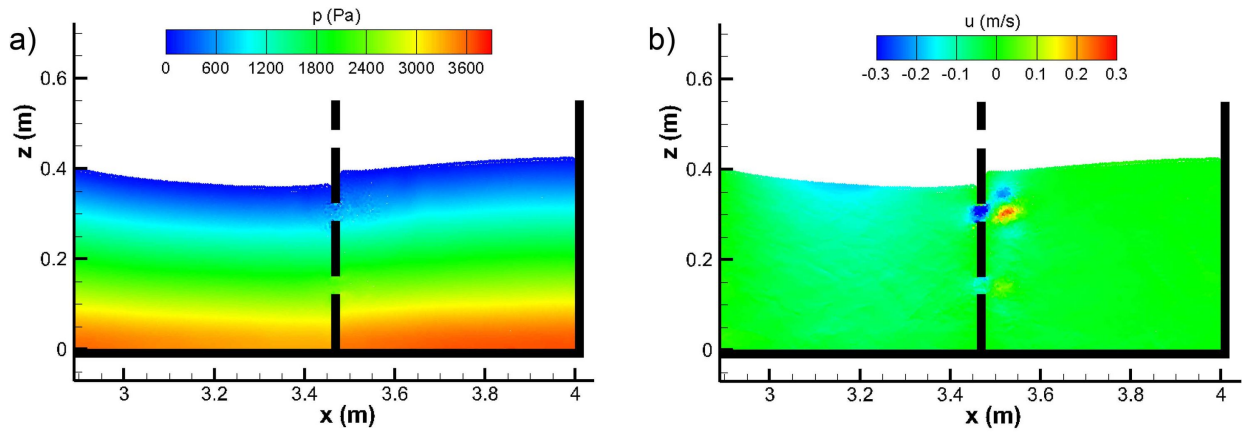

Figure 9: Frame of the SPH simulation in which the maximum dynamic pressure is encountered at the rear face in correspondence to the SWL. a) pressure field, b) horizontal velocity field.

SPH results are analyzed in terms of spatial distributions of dynamic pressures at the walls of the structure. Dynamic pressures are considered when the maximum dynamic pressure within the wave cycle appears in correspondence of the SWL, as highlighted for the front and the rear walls in Figs. 8 and 9 , respectively. The maximum dynamic pressures at the walls appear during the first wave cycle, obtained after the transition phase induced by an initial ramp function. For all the points located along the slotted and the solid walls at a mutual distance equal to $\Delta x$, the dynamic pressures are evaluated through a MLS interpolation using a Gaussian kernel with radius equal to $3 h$. The results are shown in Fig. 10, in which the hybrid diffusive SPH formulation is compared with the empirical relationship of Tabet-Aoul and Lambert (2003). This formula is based on the model by Takahashi (2002), in which the authors introduced some corrective coefficients in order to furnish a more refined distribution of dynamic pressures at a fully perforated breakwater. In the pressure diagrams, positive dynamic pressures are displayed on the external side of the walls and negative ones on their internal side. A good agreement between numerical and experimental results is obtained at the rear wall and at the front wall away from the holes. As expected, the wave loads acting on the rear wall result to be smaller than on the front wall due to wave energy dissipation induced by the slotted part of the breakwater (Tabet-Aoul and Lambert, 2003).

\section{CONCLUSIONS}

A new modeling of solid boundaries for SPH simulations has been presented. The enhancement consists of the adoption of more interpolation nodes for each considered solid particle in order to model thin structures surrounded by a fluid mass. Since the spatial resolution to model thin coastal structures such as 

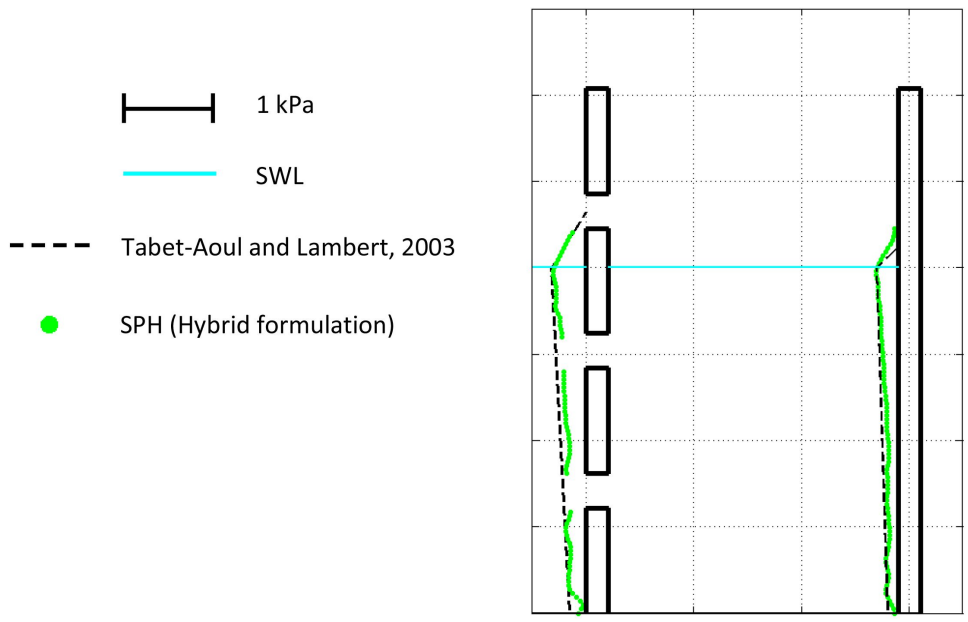

Figure 10: Spatial distribution of wave pressures at a fully perforated breakwater: comparison between SPH and the empirical solution by Tabet-Aoul and Lambert Tabet-Aoul and Lambert (2003).

decks and perforated caissons is driven by the presence of thin solid boundaries, the use of multi-node fixed ghost particles allows to avoid long, often redundant, simulations related to the standard implementation. Indeed, the one-node fixed ghost particle approach yields numerical models characterized by a number of particles which is, in 2D problems, about four times that resulting from the proposed multi-node technique. An improvement have been also introduced to well assess the pressure field by a specific diffusive term in the continuity equation considering a parameter detecting the occurrences of fluid impacts in the domain and automatically switching between two literature formulations. The SPH model has been successfully adopted to investigate the wave impact on thin coastal structures such as a horizontal deck and a fully-perforated breakwater in terms of velocity and pressure field. Further investigations will be addressed to better smooth out the high-frequency acoustic components occurring in the pressure field of the present weakly-compressible SPH solver using Wavelet Transform, as recently suggested by Meringolo et al. (2017).

\section{ACKNOWLEDGEMENTS}

We thank P. Groenenboom (ESI Group Netherlands), A. Colagrossi and S. Marrone (CNR-INSEAN) for the fruitful discussions in developing the SPH numerical strategies.

\section{References}

Antuono, M., Colagrossi, A., Marrone, S., Molteni, D., 2010. Free-surface flows solved by means of SPH schemes with numerical diffusive terms. Comp. Phys. Comm., 181, 532-549.

Antuono, M., Colagrossi, A., Marrone, S., 2012.Numerical diffusive terms in weakly-compressible SPH schemes. Comp. Phys. Comm., 183, 2570-2580.

Aristodemo, F., Federico, I., Veltri, P., Panizzo, A., 2010. Two-phase SPH modelling of advective-diffusion processes. Environ. Fluid Mech., 10, 451-470.

Aristodemo, F., Tomasicchio, G.R., Veltri, P., 2011. New model to determine forces at on-bottom slender pipelines. Coast. Eng., 58(3), 267-280.

Aristodemo, F., Meringolo, D.D., Groenenboom, P., Lo Schiavo, A., Veltri, P., Veltri, M., 2015. Assessment of dynamic pressures at vertical and perforated breakwaters through diffusive SPH schemes. Math. Probl. Eng., ID 305028, 1-10.

Aristodemo, F., Marrone, S., Federico, I., 2015. SPH modeling of plane jets into water bodies through an inflow/outflow algorithm. Ocean Eng., 105, 160-175. 
Colagrossi, A., Landrini, M., 2003. Numerical simulation of interfacial flows by smoothed particle hydrodynamics. J. Comp. Phys., 191, 448-475.

Domínguez, J.M., Crespo, A.J.C., Gómez-Gesteira, M., 2013. Optimization strategies for CPU and GPU implementations of a smoothed particle hydrodynamics method. Comp. Phys. Comm., 184(3), 617-627.

Gómez-Gesteira, M., Cerqueiro, D., Crespo, C., Dalrymple, R.A., 2005. Green water overtopping analyzed with a SPH model. Ocean Eng., 32, 223-238.

Marrone, S., Antuono, M., Colagrossi, A., Colicchio, G., Le Touzé, D., Graziani, G., 2011a. $\delta$-SPH model for simulating violent impact flows. Comput. Meth. Appl. Mech. Eng., 200, 1526-1542.

Meringolo, D.D., Aristodemo, F., Veltri, P., 2015. SPH numerical modelling of wave-perforated breakwater interaction. Coast. Eng., 101, 48-68.

Meringolo, D.D., Colagrossi, A., Marrone, S., Aristodemo, F., 2017. On the filtering of acoustic components in weakly-compressible SPH simulations. J. Fluids Struct., 70, 1-23.

Molteni, D., Colagrossi, A., 2009. A simple procedure to improve the pressure evaluation in hydrodynamic context using the SPH. Comp. Phys. Comm., 180, 861-872.

Tabet-Aoul, E.H., Lambert, E., 2003. Tentative new formula for maximum horizontal wave forces acting on perforated caisson. J. Water. Port C-ASCE, 129(1), 34-40.

Takahashi, S., 2002.Design of vertical breakwaters. Lecture note at 28th Int. Conf. on Coast. Eng., Cardiff, $1-105$. 